\title{
Adolescent Awareness of the Psychosocial Risk Factors for Depression in Selected Secondary Schools in Nairobi-Kenya
}

\author{
Ms. Priscilla Mugambi \\ Daystar University, Institute for Child Development. priscillashiku@gmail.com \\ Dr. Ciriaka Gitonga (PhD)
}

Pan Africa Christian University, P.O.Box 1672400620 Nairobi; gciriaka@pacuniversity.ac.ke

\author{
Doi:10.5901/jesr.2015.v5n3p191
}

\begin{abstract}
There are many psychosocial risk factors that lead adolescents into depression and awareness of these factors builds resilience and coping methods. The purpose of this study was to examine risk for depression among the adolescents in secondary schools in an urban setting and levels of awareness. The main objective was to examine risk factors leading to depression among adolescents. Cognitive behavioral theory was used to describe depression, its causes and solutions. The study adopted survey design and descriptive statistics were used in data analysis. A sample of 5 urban private secondary schools and a total of 130 respondents was randomly drawn. Data was collected by use of questionnaires, focus group discussions and interviews. Data was analyzed, interpreted and relevant recommendations made. The study reveals that adolescents' exposure to the conflicting relationship with parents, traumatic life experiences, peer pressure, broken relationships, and school related problems are the major psychosocial risk factors for depression in Nairobi County. A more comprehensive research is recommended to examine the extent of psychosocial risk factors for depression in Kenyan school.
\end{abstract}

Keywords: Psychosocial, Risk Factors, Depression

\section{Introduction}

Depression is defined as a mental disorder that impedes the holistic growth and development of an individual. It is defined as a disturbance in mood, thought, and body characterized by varying degrees of sadness, disappointment, loneliness, hopelessness, self-doubt, and guilt (Thapar, Stephan, DPhil, \& Thapar, 2012). Depression therefore makes it hard for a person to carry out their daily activities as it affects one physically, emotionally, socially, cognitively and spiritually. A depressed person usually has a sense of hopelessness and despair, which can lead to suicidal tendencies.

\section{Literature}

In Kenya, it is reported that depression accounts for $84 \%$ of suicides according to (Ndetei, Khasakhala, Mutiso and Mbwayo, 2010). According to Karongo (2012) the number of suicide and attempted suicide cases especially among University students has been on the increase. An earlier study by (Ndetei, Khasakhala, Mathai, \& Harder, 2008) 4\% of 12 to 17 years old and $9 \%$ of 18 to 24 years old adolescents were shown to suffer from Major Depressive Disorder (MDD). Examining secondary students (Ndetei et al. 2008) reported that out of 250 students from public secondary schools in Kenya, clinical diagnostic scores for depression were recorded in $43.7 \%$, with symptoms of depression ranging from mild to severe. According to Natembeya (2011), there had been more than 10 suicide cases reported in Murang'a County in a span of three months. Obara (2011), addressing an incident in Tetu location where four young people had committed suicide within a month, postulated that stress can progress to depression if not properly dealt with.

\subsection{Common Risk Factors for Depression in Adolescents}

Depressed adolescents are at a higher risk for abusing substance, getting recurrent depression, or other emotional and mental health problems during adulthood (Miller, Keitner, Whisman, Ryan and Epstien, 2010). Brent and Birmaher (2002) argue that, although anyone can develop depression, certain factors increase an adolescent's risk for becoming 
depressed, including; female sex, a history of depression in a parent or sibling, a prior history of depression, learning disability, a recent loss (such as, death or divorce), family problems or conflicts with parents, difficulties with friends or peers, difficulties with school work, negative outlook or poor coping skills and chronic illness. A myriad of factors then, serve as a cause while others aggravate depression.

Studies show that depression in adolescence occurs with other disorders such as eating disorders, dysthymic disorder, anxiety disorders, attention-deficit/hyperactivity disorder, oppositional defiant disorder, and substance use disorder (Shashi, Bhatia and Subhash, 2007). It is evident that depression affects the whole of a person's life, impairing academic, social, emotional and physical health, and carrying considerable stigma if no help is provided on time. The common risk factors for depression are examined below:-

\subsubsection{Psychological risk factors}

Psychological factors are those conditions that affect the mind or emotions. They affect the function of awareness, feeling, motivation, thoughts, attitudes, or other cognitive or affective characteristics of an individual that influence his or her behavior. These factors include low self-esteem, a tendency to be self-critical, perceived poor body image, feeling helpless when dealing with negative events and having relationship problems (Dryden, 2013). A study carried out by (Blackdog Institute, 2012), reported that certain personality types are prone to depressive moods than others; the anxious worrying personality, irritable, self-critical and rejection sensitive personality style, self-focused, perfectionist, the socially avoidant and the personally reserved personality style. Persons with these personality traits tend to have a low self-esteem and a pessimistic attitude.

\subsubsection{Traumatic Experiences}

According to Powers and Welsh (1999), traumatic childhood experiences such as physical abuse, negligence by caregivers, rape or death of a loved one are past and long-standing stresses that can increase chances of a person developing depressive disorder later in life. Ndetei et al. (2008) reported that, physical and sexual abuse which are highly prevalent In Kenya society, were the cause of severe depression among $10.2 \%$ young males and $6.9 \%$ young females. Cases of physical and sexual abuse are evident in both low and high economic status and especially among adolescents. When abused children cannot run away from their suffering physically, they will try to do it psychologically through depression, withdrawal and hysteria (Mwiti, 2006).

\subsubsection{Peer Relationship}

Teenagers tend to socialize more with their peers and regard the opinions of their peers more highly. According to Erikson (1968), the crisis of adolescence stage involves a struggle to define one's self-identity while still fitting in and being accepted and thus a risk to being influenced negatively. Teenagers are overly sensitive to the way peers treat them, thus rejection by peers of the same or opposite sex may lead to depression. They also tend to seek advice and comfort from their peers who may mislead them due to their lack of understanding and experience. The need to be independent and craving for peer approval makes the adolescent vulnerable to developing unhealthy coping mechanisms in stressful situation (Saluja, lachan and Scheidt 2004). Lewis, 1992, reported that this causes significant stress. If they lack good appraisal or get rejected by peers they tend to develop a low self-esteem or get into deviant behavior as a way of coping.

\subsubsection{Cultural factors}

Cultural change experienced today has significant effects on the way adolescents relate with the parents and other member of the society. The change in technological innovations, ideologies, cultural conflict and planned change (Kibera and Kimokoti, 2007) accounts for increased levels of stress among adolescents. This has led to increase access to information and reduce interactions between adults and young people. It is reported that an adolescent spends more than two-thirds of their time on the Internet in the urban centers. These factors influence adolescents' behavior making them live in a world of fantasies, with unrealistic ambitions and failure to keep up with peers may lead to a low selfesteem. 


\subsubsection{Parent-child relationship}

Every parent wants to bring up successful children yet different societies and generations define success differently. Parents need to understand how they may be contributing to the depression state of their children so that they can change any negative influence. A study by Ndetei et al. (2008) shows that family dysfunctions are a great source of psychiatric disorders in children. In dysfunctional families, there is a high prevalence of parent-to-parent or parent-tochild conflicts, these increases the risk of adolescence developing psychiatric disorders. The study further shows that the uninvolved parenting behavior is associated with child negligence, both physically and emotionally. Parenting styles pose as a risk factor for depression, mothers who are authoritarian emotionally and physically abused their children, whereas the mothers who are uninvolved, physically and emotionally neglected their children. According to Powers and Welsh (1999) an uncaring parent or an abusive parent is a great past stressor to the growth and development of a child. Being neglected by a parent may cause a child to develop low self-esteem and therefore becomes quiet vulnerable to developing depressive disorders in adulthood. The mental status of the parent usually affects their parenting style and can make children internalize negative aspects of their life giving no room to focusing on their strengths. The teenagers of uncaring parents lack a healthy avenue to express their feelings and thus they quietly internalize them or may seek to relieve their tension through ways, which consequently lead to or worsen the depressive symptoms.

The quality of the parents' marriage, parenting style, the emotional status and role modeling of the parents are related to depression in adolescents (Gurian, 2012). Parent's negative emotions can lead, to negative outcomes in adolescent development due to creation of problematic parenting behavior. An adolescent with deviant behavior will consequently cause stress to the parents, leading to more hostility and this cycle will eventually impact negatively on the whole family system and its cohesion.

\subsubsection{Academic Environment Risk Factors}

Unrealistic academic expectations can create a great sense of rejection and therefore lead to deep disappointment. It was reported that two students had committed suicide after the release of exam results, allegedly because of the failure in exams. According to Cantwell (2007) while teens in the United States engage more in sports as an after school activity, Kenyan teens are encouraged to focus more on academics than sports. Todays modernized generation puts a lot of emphasis on academic success. Irrational belief may make a teenager believe that failure in examination means failure in life. This would lead to great distress and the fear of failure may make even a gifted student to fail in exams.

\subsection{Adolescents' awareness on depression}

Increased knowledge and awareness signs and symptoms of depression among adolescents is essential in preventing depression. Many adolescents are unsure why they are depressed (Bonin, 2004). While the cause of some disease or disorder are well known, depression still startles the researchers due to the fact that it is not clear how and when it comes. There are some good ideas about what some of the pressures or strains that result in depression are, but they are not all agreed upon and there might be others (Clark, Jansen, \& Cloy, 2012). This then makes early detection of depression difficult since there is no one definite cause. Deborah (2013), noted that diagnosis of depression in children and adolescents just as with adult depression, is not as clear-cut as it is for other ailments. There is no test to diagnose depression. Due to the complexity of depression many adolescents suffer from depression without realizing what they are suffering from. The situation is worsened when those around them label them lazy or defiant due to their strange behavior as they seek to cope with the disturbed mood.

According to Weiten, (1995), when adolescents fail to cope with the adolescence crisis they may end up becoming depressed, abuse drugs and alcohol or indulge in chronic delinquency and crime. Adolescent crisis may be worsened by traumatic or bad events in an adolescent's life. Influence of bad events is a major cause of depression among adolescents. Examples of bad events occurring in a teenager's life are; academic failures, bullying, rejection from peers or friends of the opposite sex, conflict with parents, bad parental relationships or the death of a close person (Jung, 2013).

\subsection{Effects of depression on the adolescent development}

Depression is expressed differently within different individuals and in the various developmental stages. This may be due 
to the social environment, the precipitating factors and individual temperaments. According to Conrad, Flay, and Hill (1992), rebellious attitudes and unhealthy behaviors in teenagers may actually be an indication of depression. Depressed adolescents tend to develop coping mechanisms as a way of seeking comfort and remedy to their recurrent discomforts. Teenagers are likely to engage in high-risk behaviors and dangerous actions such as; out-of-control drinking, reckless driving and unsafe sex. Mbaya (2009) explains that, adolescents respond to stress through misuse of substance. People, who misuse substance; alcohol and other drugs of addictions, do so to escape the pain and challenges of life. Depression has been shown to be associated with youth suicidal behavior, which is a major problem in many countries, as it is the third leading cause of death in young people.

\subsection{Theoretical Framework: Cognitive Behavioral Perspectives of Depression}

Cognitive behavioral theory states that it is important to examine the risk factors for depression, factors that maintain it and the therapies used in its treatment. From a cognitive perspective, depression is a result of irrational thinking about oneself, one's current life situation, or one's future. Depression therefore results from faulty, negative, maladaptive, or irrational cognitions, which take the form of distorted thoughts and judgments. Depressed people are pessimistic and therefore, usually view themselves, their environment, and the future in a negative way (Beck, 1987). As a result, they usually misinterpret facts in negative ways and tend to blame themselves for any misfortune that occurs. Situations are viewed as being worse than they are. Such people do not seem to see anything good coming out of their life and this leads to hopelessness which can lead to the risk taking behaviors especially, seen in depressed adolescents.

Bradshaw (1987) noted that particular failures of information processing characterize a depressed mind. He further explains that depressed people will tend to consider the negative information that appeals to their negative expectations, and selectively ignore information, which is against those expectations. Therefore depressed persons have a negative worldview where most aspects of life are interpreted based on the worst information. This reflects a pessimistic personality.

\section{Methodology}

This study adopted descriptive survey design. Mugenda and Mugenda (2003) states that descriptive design portrays the facts as they really are, that is if another researcher goes to conduct the same study, he or she will find the situation as it was described. The study area was Westland's division in Nairobi County. Westlands division is located $3.1 \mathrm{~km}$, northwest of the central business center of Nairobi (Distant map, 2013). Its diverse context makes it a good representative of adolescents from different Kenyan ethnic groups and from high social and economic status thus offering a good representation of the national culture. Nairobi County was ideal for this study because it is cosmopolitan in nature and therefore can allow generalization of findings to other major cities in Africa. The study population was the adolescent students in the private secondary schools of Nairobi County. There are a total of 35 registered PSS in Westlands division with a total of 6,761 students (EMIS Unit, MOE, 2007). The sample was drawn from 5 schools of various categories out of the 35 schools in Westlands division. The different categories of schools were used so as to obtain varied context for adolescents' depression risk factors. The data collection tools used in the study included; the questionnaires for students, focus group discussions for the selected students and in-depth interviews for the staff. A Likert scale questionnaire was administered to the respondents. The questionnaire had some contingency questions whose purpose was to measure the preference of psychosocial risk factors for depression, describe the experiences and lead to probing for more information. The focus group discussion guide was used in obtaining qualitative data pertaining to the adolescents' responses to and effects of the risk factors for depression in their lives, as well as to get their perspectives and life experiences on the same.

\section{Results and Discussions}

Various psychosocial risk factors for depression among adolescents were examined. They included risk factors associated with: the psychological factors, traumatic experiences, peer relationships, parent-child relationships, cultural factors and academic environment. The respondents were asked to rate the factors that served as a cause of stress in their lives. From the likert scale the scores of strongly disagree and disagree were combined and interpreted as disagreeing or negating while those of agree and strongly agree were combined and interpreted as affirming, agreeing or concurring with the stated statements and factors. 


\subsection{Demographic data}

The respondents were in the adolescence stage of development, with the majority being in their mid-adolescence stage with results showing; $30 \%$ were 16 years' old, $23.8 \%$ were 17 years, $16.9 \%$ were 18 years, $5.4 \%$ were 14 years and $3.8 \%$ were 13 years old. There were, $63.8 \%$ female and $34.6 \%$ male. This implies that most of the respondents were female.

\subsection{Results of the psychosocial risk factors for depression}

\subsubsection{Traumatic experiences as Risk Factor for Depression}

The results reveal that $80 \%$ of the respondents had experienced a traumatic event, which led to varying degrees of depressive moods. Only $18.4 \%$ reported that they had not experienced a traumatic event. This shows that the majority of the teenagers had an experience that was considered traumatic. The common traumatic events recorded was sexual harassment and rape, death of a parent or a loved one, being involved in or witnessing an accident, disabilities and sickness, separation and divorce of parents, broken relationship, being rejected by friends and being bullied among others. Ndetei et al. (2008) reported that physical and sexual abuse was highly prevalent among the youths and it is related to severe depression.

\subsubsection{Parent-child relationship}

The results reveal that $76.9 \%$ of the respondents perceived their parents-child relationship as a cause of stress in their lives as compared to $22.3 \%$ who disagreed. One respondent stated "my parents kept blaming me for failure in exams yet the reason for failing was lack of concentration due to constantly thinking about my alcoholic father". This implies that majority of the respondents felt that the nature of their relationship was affecting them psychologically. The findings are supported by (Ndetei et. al., 2008) who found that children in dysfunctional families were highly vulnerable to developing a psychiatric disorder.

Table 1: Parent-Child relationships

\begin{tabular}{lc}
\hline & Percent \\
\hline Strongly disagree & 14.6 \\
Disagree & 7.7 \\
Agree & 39.2 \\
Strongly agree & 37.7 \\
No response & 0.8 \\
\hline Total & 100 \\
\hline
\end{tabular}

The result further reveal that $84.6 \%$ of respondent felt that being misunderstood by parents was a cause of stress in their lives, while $15.4 \%$ stated that they had a good relationship with their parents. According to Lewis (1992), although adolescents are in a stage where they need freedom and autonomy they are still dependent on parents for their basic needs. Adolescents conflict with their parents as they seek independence and experiment dangerous behaviors. This agrees with the findings as the teenagers described their parents as being; very strict and over-protective. They felt that their parents' did not relate with the things they went through and therefore they were not supportive. The parents were also viewed as suspicious and judgmental.

\subsubsection{Peer relationships as Risk Factor for Depression}

Majority of the respondents $61.6 \%$ felt that being rejected by friends was a major source of stress in their lives. In this study $37.7 \%$ did not perceive peer relationships as a threat for they felt they were able to cope well. Saluja et al. (2004) explains that, in search of identity, adolescents struggle to find a place in society, to be recognized and to be accepted by their peers. Adolescents are faced with moral dilemma, where they feel confused about the moral decisions that must be taken. This implies that the respondents being in the adolescent stage of development were struggling to be recognized and accepted by peers and failure in this as well as the conflicting moral dilemma served as a risk factor for depression. 
Table 2: Peer Relationship is Important

\begin{tabular}{lc}
\hline & Percent \\
\hline Strongly disagree & 21.5 \\
Disagree & 16.2 \\
Agree & 36.2 \\
Strongly agree & 25.4 \\
No response & 0.8 \\
\hline Total & 100 \\
\hline
\end{tabular}

Broken relationships and rejection by peers affects the teenagers psychologically since relationships give the adolescent's psychological satisfaction as they are highly self-conscious and in need of peers to define their identity (Lewis, 1992). The findings concurs with Jung (2013), that the Influence of bad events is a major cause of depression among adolescents. The examples of bad events occurring in a teenager's life are; academic failures, bullying, rejection from peers or friends of the opposite sex, conflict with parents, bad parental relationships or the death of a close person (Jung, 2013).

The teachers in this study felt that family related issues and particularly parent-child relationship significantly affected the respondents. The data reveal that $36.4 \%$ parenting relationship was significant risk factor, $27.3 \%$ school life and exams was a risk factor, $27.3 \%$ traumatic life experiences, while only $9.1 \%$ stated self-esteem and peer influence was risk factor for depression. According to Powers and Welsh (1999) the most clearly established past stressor is that of an uncaring parent or an abusive parent. Hurlock (1991) postulates that, the changes that occur in adolescence which may affect the relationship between parent and teenager, are; puberty, development of logical reasoning, idealistic ideas that rise, expectations that are not met, changes at school, peers, friendships, dating, and relationships to freedom. This is in agreement with the findings.

From the focus group discussion the respondents felt that various situations, circumstances or events made them susceptible to depression. They reported that lack of support and conflict with parents was rated highly together with peer pressure and use of drugs, Accidents, death and disabilities comprised of $17.1 \%$ while broken relationships and rejection by peers was $14.6 \%$, Physical and sexual harassment $12.2 \%$ and being expelled from school comprised of $4.9 \%$. This indicates that the greatest fears for teenagers are lack of support and conflict with parents as well as peer pressure and drug abuse. This concurs with Brent and Birmaher (2002) that, among the factors that increase an adolescent's risk for becoming depressed includes; a history of depression in a parent or sibling, a prior history of depression, learning disability, a recent loss (such as, death or divorce), family problems or conflicts with parents, difficulties with friends or peers, difficulties with school work, negative outlook or poor coping skills and chronic illness.

\subsubsection{Academic Environment Risk Factors for Depression}

More than half of the respondents $58.8 \%$ stated that academic life was a cause of stress. In this study $40.7 \%$ stated that they were able to cope with school related pressure. This implies that majority of the students were stressed by academic life. This supports Goldman (1994) explanation that, pressure from school competition creates a great deal of tension and that parental pressure on academic performance can be a cause of depression among teens.

Table 3: The Academic Environment and Risk Factors for Depression

\begin{tabular}{lc}
\hline & Percent \\
\hline Strongly disagree & 16.9 \\
Disagree & 23.8 \\
Agree & 37.7 \\
Strongly agree & 20.8 \\
No response & 0.8 \\
\hline Total & 100 \\
\hline
\end{tabular}

\subsection{Gender Difference in Risk Factors}

The female respondents were at a higher risk $31.20 \%$ of getting depression as compared to the male respondents $18.20 \%$. Brent and Birmaher, (2002) includes being of female sex as one of the factors that increase the risk of being depressed. 
Table 4: Gender as a risk factor for depression

\begin{tabular}{lcccc}
\hline Gender of students & Low risk & Medium risk & High risk & Total \\
\hline Male & $38.60 \%$ & $43.20 \%$ & $18.20 \%$ & $100.00 \%$ \\
Female & $25.00 \%$ & $43.80 \%$ & $31.20 \%$ & $100.00 \%$ \\
\hline Total & $29.80 \%$ & $43.50 \%$ & $26.60 \%$ & $100.00 \%$ \\
\hline
\end{tabular}

The 16 years old were at the highest risk (28.2\%) of suffering from depression, while the 17 years and older followed closely at $27.3 \%$. the majority at the lowest risk were 13 to 15 years old. Therefore the younger teenagers were at a low risk level while the older ones were at a higher risk. The majority of the respondents were at the medium level (43.20\%) of being at risk of depression. A study by Gladstone, Beardslee and O'Connor, (2011), indicated that $50 \%$ of adult disorders have an onset by age 14 and that the early symptoms of depression emerge a few years before full diagnostic criteria are met with symptoms emerging 3 to 4 years earlier before the diagnosis. The findings agree with this in that, at an early age the risk factors for depression may not be clearly defined.

Table 5: Age as risk factor for depression

\begin{tabular}{lcccc}
\hline Age of respondents in categories & Low risk & Medium risk & High risk & Total \\
\hline 13 to 15 years & $41.90 \%$ & $35.50 \%$ & $22.60 \%$ & $100.00 \%$ \\
16 years & $25.60 \%$ & $46.20 \%$ & $28.20 \%$ & $100.00 \%$ \\
17 years or older & $27.30 \%$ & $45.50 \%$ & $27.30 \%$ & $100.00 \%$ \\
\hline Total & $30.40 \%$ & $43.20 \%$ & $26.40 \%$ & $100.00 \%$ \\
\hline
\end{tabular}

\subsection{Adolescent Awareness of Risk Factors for Depression}

During adolescent stage of development, abstract reasoning and perspective-taking abilities increase. As compared with younger children, early adolescents are better able to reflect on their beliefs and to engage in hypothesis testing by examining evidence and considering alternatives (Gillham, 2009). The level of awareness on risk factors for depression was thus crucial in determining the risk or resilience of the teenagers.

Table 6: Awareness of Depressive Moods

\begin{tabular}{lc}
\hline & Percent \\
\hline Strongly disagree & 3.1 \\
Disagree & 3.1 \\
Agree & 30 \\
Strongly agree & 63.1 \\
No response & 0.8 \\
\hline Total & 100 \\
\hline
\end{tabular}

To assess the level of awareness the respondents' perspectives were sought in relation to their response on the statements that were related to the risk factors for depression. Most of the respondent was aware of depressive moods 93.1\% but felt that that problem are part of life for every person with only 9.2\% feeling otherwise. This indicates that majority of the adolescents were well aware that problems are inevitable in life. According to Kohut, Cooper, Nickolaus, Russell, \& Cunnick, (2002). optimism improves the immune system, prevents chronic disease, and it helps people cope with unfortunate news. People who are optimistic are happier, receive more social support, are less stressed, and are less depressed.

\subsection{Awareness of risk associated with the academics environment}

Majority of the respondents, $89.2 \%$ were aware of the challenges associated with academics and felt they could do something about it with $8.5 \%$ expressing feelings of helplessness. Those who felt that nothing could be done imply a sense of despair which could have been as result of constantly failure in exams. The majority of the respondents had rational thought, which serves as resilient factor for depression. According to Jung (2013), what makes a bad event a real 
problem is the attitude. If a bad event were faced positively, it would not contribute to depression.

Table 7: I can do something about my academics

\begin{tabular}{lc}
\hline & Percent \\
\hline Strongly disagree & 64.6 \\
Disagree & 24.6 \\
Agree & 0.8 \\
Strongly agree & 7.7 \\
No response & 2.3 \\
\hline Total & 100 \\
\hline
\end{tabular}

The results shows that $58.5 \%$ were often aware and felt they could do something but $40.7 \%$ felt they needed support to be able to do something about their academics. This indicates that majority of the teenagers were able to recognize negative emotions in the academic experiences. Cognitive behavioral theorists' suggest that, by giving evidence against unhelpful thoughts and adopting more realistic and balanced perspectives over a situation one can help replace negative emotions with positive ones. Depressed people have negative emotions and therefore they tend to view themselves, their environment, and the future in a negative, pessimistic light (Beck, 1987).

Table 8: I Feel Like I can do nothing about my academics

\begin{tabular}{lc}
\hline & Percent \\
\hline Strongly disagree & 64.6 \\
Disagree & 24.6 \\
Agree & 0.8 \\
Strongly agree & 7.7 \\
No response & 2.3 \\
\hline Total & 100 \\
\hline
\end{tabular}

\subsection{Gender differences and awareness levels}

The results reveal that male respondents had a higher level of self-awareness as compared to the female respondents. This indicates that most of the female respondents were not aware that the feelings of sadness and irrational thoughts were risk factor for depression.

Table 9: Gender and level of self-awareness

\begin{tabular}{lccc}
\hline Gender of students & Low awareness & Medium awareness & High awareness \\
\hline Male & $35.60 \%$ & $33.30 \%$ & $31.10 \%$ \\
Female & $33.80 \%$ & $37.70 \%$ & $28.60 \%$ \\
\hline Total & $34.40 \%$ & $36.10 \%$ & $29.50 \%$ \\
\hline
\end{tabular}

\subsection{Age differences and awareness levels}

The majority of the respondents at the high level of awareness on the risk factors for depression were aged 13 to 15 years' old, 16 year old were next at (29.7\%) and the least level of awareness was on the 17 years and older (24.10\%). At the lowest level were both the 16 years and those above 17 years old (37\%). The total averages show that there was a high level of awareness, which indicates that the majority of teenagers were resilient to depression. A good level of selfawareness is likely to create resilience against depression as one gets to use their will power to avoid irrational thoughts. Resilience is a dynamic process encompassing the manifestation of positive functioning despite possessing vulnerabilities or the presence of high risk Gerald, Haeffel, Elena \& Grigorenko, (2007). Resilience may be the process of, capacity for, or outcome of successful adaptation despite challenging and threatening circumstances. From the findings the majority of the respondents felt that they could do something about their lack of interest in academics. This implies that they believed they should not give up and let their desires control them. 
Table 10: Age and level of awareness

\begin{tabular}{lccc}
\hline Age of respondents in categories & Low awareness & Medium awareness & High awareness \\
\hline 13 to 15 years & $28.10 \%$ & $34.40 \%$ & $37.50 \%$ \\
16 years & $37.80 \%$ & $32.40 \%$ & $29.70 \%$ \\
17 years or older & $37.00 \%$ & $38.90 \%$ & $24.10 \%$ \\
\hline Total & $35.00 \%$ & $35.80 \%$ & $29.30 \%$ \\
\hline
\end{tabular}

\section{Conclusion and Discussions}

The results reveal that majority of the high school students were at risk of suffering from depression. The results show the following as the major risk factors; being misunderstood by parents (84\%), the traumatic life experiences (80\%), parents' unstable relationship (76.9\%), rejection by friends (61.6\%), school and academic related problems (58.8\%), pessimistic feelings, that significant people don't care about them (57.7\%), feeling that they are not as good as other people (54.7\%), their unhappy childhood experiences (51.48\%) and the addictions (48.4\%). These findings agree with Powers and Welsh (1999) that the most clearly established past stressor is that of an uncaring parent or an abusive parent. The findings are in line with a study by Ndetei et al. (2008), that family dysfunctions are mediating factors for children to develop psychiatric disorders.

Majority of the respondents $80 \%$ attributed the source of stress in their life to traumatic experiences as reported by Brent and Birmaher (2002). More than half of the respondents had irrational thoughts of feeling not cared for (57.7\%) and not as good as other people (54.7\%) this reflects a pessimistic attitude. The respondents were found to have a high level of awareness, which serves as a resilient factor. The majority, 93.1\% affirmed that problems are part of life for every person and this indicates that the teenagers were optimistic about life. Majority of the teenagers (89.2) were aware that they could improve on their academics. This indicates that only a few (8.5\%) of them were likely to despair in life due to academic failure. The majority (71.6\%) were aware that they had control over their emotions of being sad or happy.

Based on the findings in this study there is need to carry out a more comprehensive study in Kenya schools to determine the psychosocial risk factors for depression in adolescents with the purpose of developing intervention policies.

\section{References}

American Psychiatric Association. (2013). Diagnostic and Statistical Manual of mental Disorders. (5th ed), (DSM-5). Washington DC: American Psychiatric Association.

Beck, A. T. (1987). Cognitive models of depression. Journal of Cognitive Psychotherapy. 1987; 1:5-37.

Blackdog Institute (2012). Causes of depression. Retrieved July 18, 2013, from http://www.blackdoginstitute.org.au/public/depression/ causesofdepression/index.cfm.

Bonin, L. (2004). Patient information: Depression in adolescents (Beyond the Basics). Retrieved July 21, 2013, from http://www. Risk factors/Depression in adolescents.htm

Bradshaw, J. (1987). The family: A revolutionary way of self-discovery: Pompano Beach, Fla.: Health Communications.

Brent, D. A., \& Birmaher, B. (2002). Clinical practice. Adolescent depression. A journal of N Engl J med 2002; 347:667

Chege, A. W., (2012) an investigation of peer influence on deviant behavior in secondary schools in Nairobi County. Retrieved November 18, 2013, from http://erepository.uonbi.ac.ke/handle/123456789/8073.

Conrad, K. M., Flay, B. R., \& Hill, D. (1992). Why children start smoking cigarettes: predictors of onset. British Journal of Addiction. 1992; 87(12):1711-24.

Clark, S., Jansen, L., \& Cloy, A. (2012). Treatment of childhood and adolescent depression. Retrieved October 6, 2013, from http://umm.edu/health/medical/reports/articles/depressionz3334/YFP

Deborah, (2013). Depression in Children: Symptoms, Causes, Diagnosis and Treatment. Retrieved on September 2, 2013, from http://depressioninchildren.com/symptoms-causes/

Dryden, E. R. (2013). Teen Depression. Medicinenet. Retrieved July 23, 2013, from http://www.medicinenet.com/teen_depression larticle.htm

Erikson, E. H. (1968). Identity, youth and crisis. New York: W. W. Norton Company,

Gerald, J., Haeffel, Elena L., \& Grigorenko, (2007). Cognitive Vulnerability to Depression: Exploring Risk and Resilience. Journal of Child Adolescent Psychiatric Clin N Am16 435-448. New Haven, CT 06520, USA.

Gillham, J. (2009). Preventing Teen Depression A Program Based on Cognitive Behavioral Therapy Gives Adolescents Some Tools to Stave Off Depression. Retrieved February 2, 2014, from http://media.swarthmore.edu/bulletin/?p=295.

Gladstone, T. R., Beardslee, W. R., \& O'Connor, E. E. (2011). The Prevention of Adolescent Depression. Journal of Psychiatric Clin North Am. 2011 March; 34(1): 35-52. Retrieved January 18, 2014, from http// theses materials/depression thesis/The 
Prevention of Adolescent Depression.htm\#R6

Goldman, N. (1994). Life issues: Emotional disorders. New York: Marshall Cavendish.

Gurian, A. (2012). Depression in Adolescence: The child study center. Retrieved on January 22, 2014, from http://www.aboutourkids. org/articles/depression_in_adolescence_does_gender_matter

Hurlock, E.B. (1991). Adolescent Development. New York: MčGraw Hill Book Company.

Jung C. G, (2013). Depression in teens: a scary problem with an easy solution. Retrieved on October 22, 2013, from http://depression teenshelp.com/depression-with-teenagers-reasons/

Karongo C. (2012). Suicide: Parents need to wake up. Retrieved on January 28, 2014 from http://www.capitalfm.co.ke/news/2012/07 /suicide-parents-need-to-wake-up/? wpmp_switcher=mobile

Khasakhala, L. I., Ndetei, D. M., Mutiso. V., Mbwayo, A. W., \& Mathai M. (2012). The prevalence of depression among adolescents in Nairobi public secondary schools: Associated with perceived maladaptive parenting behavior. African journal of psychiatry. . Retrieved November 22, from http://www.annals-general-psychiatry.com/content/12/1/13

Kibera, L. W., \& Kimokoti, A. (2007). Fundamentals of sociology of education: With reference to Africa. Nairobi: University of Nairobi Press.

Kohut, Cooper, Nickolaus, Russell, \& Cunnick, (2002). Exercise and psychosocial factors modulate immunity to influenza vaccine in elderly individuals. Journal of gerontology, Ageing, Biological science and medical science, 57,M557-62).

Langholt, A (2013) Common Causes of Peer Pressure in Teens. Retrieved February 2, 2014, from: http://www.life123.com/parenting/ tweens-teens/peer-pressure/causes-of-peer-pressure.shtml.

Lewis, M. (1992). Shame, The exposed self. New York: The Free Press

Linda MacKay, L. (2012). The Family Systems Institute. Neutral Bay, Sydney.

Mamah, D., Mbwayo, A., Mutiso, V., Barch, D., Constantino, J., Nsofo T., Khasakhala, L., \& Ndetei D. (2011). A Survey of PsychosisRisk Symptoms in Kenya. Retrieved September 6, 2013 from http://www.ncbi.nlm.nih.gov/pmc/articles/PMC3265657/

Mbaya, M. (2009). Bringing up positive teenagers in a negative world: With solid proven principles. Kenya: Flame keeper publishing company.

Mental Health America, (2013). Depression in teens. Retrieved November 20, 2013, from http://www.mentalhealthamerica.net/ conditions/depression-teens

Miller, I. W., Keitner, G., I., Whisman, A., Ryan, C. E., \& Epstein, B. (2010). Depressed patients with dysfunctional families: description and course of illness.

Natembeya, (2011). Muranga in shock: Three commit suicide. The Star, September 1, 2011. Retrieved January 22, 2014 from http://www. Murang'a in shock as three commit suicide _ The Star.htm

National Research Council and Institute of Medicine (NRCIM), (2009). Preventing emotional and behavioral disorders among young people: progress and possibilities. National Academies Press; 2009

Ndetei, D. M., Khasakhala, L. I., Mutiso, V. \& Mbwayo, A. W. (2010). Sociality and depression among adult patients admitted in general medical facilities in Kenya. Annals of general psychiatry 2010, 9:7.

Ndetei, D. M., Khasakhala, L. I., Nyabola, L., Ongecha F. O., Seedat S., Mutiso, V., Kokonya. D. \& Odhiambo G. (2008). The prevalence of depression and anxiety symptoms and syndromes in Kenyan children and adolescents. Journal of child and adolescent mental health 2008, 20(1):33-51

Ndetei, D., M., Khasakhala, L., I., Mathai, M. \& Harder, V. (2008). Major depressive disorder in a Kenyan youth sample: relationship with parenting behavior and parental psychiatric disorders. Africa Mental Health Foundation. Retrieved on July 5, 2013, from http://www.annals-general-psychiatry.com/content/12/1/15.

Obara, (2011). Three commit suicide. The Star, September 1, 2011. Retrieved January 22, 2014 from http://www. murang'a in shock as three commit suicide_the Star.htm

Page, D. J. (2006). Abnormal psychology: A clinical approach to psychological deviants. McGraw Hill: New Delhi.

Powers, S. \& Welsh, D., P. (1999). Mother-daughter interactions and adolescent girls' depression. In Conflict and Cohesion in Families. Mahwah, NJ: Lawrence Erlbaum Publishers. pp. 243-281.

Saluja, G., Iachan, R. \& Scheidt, P.,C. (2004). Prevalence of and risk factors for depressive symptoms among young adolescents. Arch Pediatric Adolescent Med; 158:760.

Shashi, K. Bhatia, M., \& Subhash, C. (2007). Childhood and adolescent depression. Journal of American family physician.am fam physician 2007 Jan 1;75(1).

Thapar, A., Stephan, C., DPhil, \& Thapar, K., (2012). Depression in Adolescents. Retrieved July 21, 2013, from http://www.ncbi.nlm.nih. gov/pmc/articles/PMC3488279/.

Weiten, W. (1995). Psychology: Themes and variations. Pacific Grove: Brooks/Cole. 